\title{
Highly Efficient Noise-Assisted Energy Transport in Classical Oscillator Systems
}

\author{
R. de J. León-Montiel ${ }^{1, *}$ and Juan P. Torres ${ }^{1,2, \dagger}$ \\ ${ }^{1}$ ICFO-Institut de Ciencies Fotoniques, Mediterranean Technology Park, 08860 Castelldefels (Barcelona), Spain \\ ${ }^{2}$ Department of Signal Theory and Communications, Campus Nord D3, Universitat Politecnica de Catalunya, 08034 Barcelona, Spain
}

(Received 7 January 2013; published 21 May 2013)

\begin{abstract}
Photosynthesis is a biological process that involves the highly efficient transport of energy captured from the Sun to a reaction center, where conversion into useful biochemical energy takes place. Using a quantum description, Rebentrost et al. [New J. Phys. 11, 033003 (2009)] and Plenio and Huelga [New J. Phys. 10, 113019 (2008)] have explained this high efficiency as the result of the interplay between the quantum coherent evolution of the photosynthetic system and noise introduced by its surrounding environment. Even though one can always use a quantum perspective to describe any physical process, since everything follows the laws of quantum mechanics, is the use of quantum theory imperative to explain this high efficiency? Recently, it has been shown by Eisfeld and Briggs [Phys. Rev. E 85, 046118 (2012)] that a purely classical model can be used to explain main aspects of the energy transfer in photosynthetic systems. Using this approach, we demonstrate explicitly here that highly efficient noiseassisted energy transport can be found as well in purely classical systems. The wider scope of applicability of the enhancement of energy transfer assisted by noise might open new ways for developing new technologies aimed at enhancing the efficiency of a myriad of energy transfer systems, from information channels in microelectronic circuits to long-distance high-voltage electrical lines.
\end{abstract}

DOI: 10.1103/PhysRevLett.110.218101

PACS numbers: 87.10.-e, 05.40.Ca, 82.20.Nk, 82.20.Rp

Because of its undoubted importance for all life on Earth, molecular mechanisms of energy transport in photosynthetic light-harvesting complexes have been a subject of study for decades [1-4]. In recent years, a renewed interest on this topic has arisen [5,6], mainly due to the unexpected observation of long-lived electronic coherences in the energy transfer process of photosynthetic systems, particularly in the nowadays most widely investigated system, the Fenna-Matthews-Olson (FMO) complex [7-9].

As a consequence of these findings, several theoretical studies have been devoted to describing how coherence effects in a quantum scenario might play an important role in the remarkably high efficiency of energy transfer in photosynthetic systems [10-12]. This is especially notable since it takes place in a scenario apparently not propitious for the observation of quantum effects. In particular, it has been suggested that high efficiency transport arises as a result of the dynamical interplay between the quantum coherent evolution of the photosynthetic system and the dephasing noise introduced by its surrounding environment, a phenomenon called environment-assisted quantum transport (ENAQT) [13] or dephasing-assisted energy transport [14].

As stated in Ref. [15], ENAQT can be understood as the suppression of coherent quantum localization mediated by noise, helping the excitation to move faster through the photosynthetic system, thus increasing the efficiency of energy transport. In this way, ENAQT might be seen as a phenomenon that exists only in a regime where the quantum and classical worlds overlap. Notwithstanding, making use of the quantum-classical correspondence of electronic energy transfer presented in Ref. [16], we show here that the same effect can also be found in purely classical systems. Our departure point is based on the consideration that aggregates of coupled monomers (such as the FMO complex) can also be described as a system of weakly interacting classical oscillators [17]. We then demonstrate that the noise-assisted enhancement of transport efficiency in the FMO complex, shown in Refs. [13,14], and based on a pure quantum formalism, can also be found in a purely classical model, without the need to resort to quantum effects.

For the sake of comparison and clarity, we will first model the FMO complex as a quantum system of $N$ interacting sites, where the interaction of each site with its surrounding environment is modeled by a pure dephasing process. We have adopted this model because of its extended use for describing noise-assisted energy transfer processes in photosynthetic systems [13,14]. Next, we will present the classical model of Refs. [16,17], which corresponds to a system of $N$ weakly coupled harmonic classical oscillators. In this case, environmental effects are introduced by assuming that the frequency of each oscillator varies stochastically as a Gaussian Markov process. Finally, we will solve both models using the site energies and coupling coefficients for the FMO complex of Prosthecochloris aestuarii to show that the same environment-assisted energy transfer effect can be found in both classical and quantum models.

The Hamiltonian of a system comprising $N$ interacting sites in the presence of a single excitation is given by 


$$
\hat{H}_{S}=\sum_{n=1}^{N} \epsilon_{n}|n\rangle\left\langle n\left|+\sum_{n \neq m}^{N} V_{n m}\right| n\right\rangle\langle m|,
$$

where $|n\rangle$ denotes the excitation being at site $n$. The $n$ th-site energies and the coupling between sites $n$ and $m$ are described by $\epsilon_{n}$ and $V_{n m}$, respectively.

We make use of a simple model where the dynamics of the system interacting with a surrounding environment is described by a Lindblad master equation, which in the Born-Markov and secular approximations is written as [18]

$$
\frac{\partial \hat{\rho}_{n m}}{\partial t}=-\frac{i}{\hbar}\left[\hat{H}_{S}, \hat{\rho}\right]_{n m}+\hat{\mathcal{L}}_{\mathrm{deph}}[\hat{\rho}]_{n m}+\hat{D}[\hat{\rho}]_{n m} .
$$

Here, the interaction of the system with the environment is characterized by a pure dephasing process given by the Lindblad operator $\hat{\mathcal{L}}_{\text {deph }}[\hat{\rho}]_{n m}=-\left[1 / 2\left(\gamma_{n}+\gamma_{m}\right)-\right.$ $\left.\sqrt{\gamma_{n} \gamma_{m}} \delta_{n m}\right] \hat{\rho}_{n m}$, with $\gamma_{n}$ being the dephasing rates. Although the pure dephasing model is not able to capture important aspects of electronic energy transfer, such as phonon relaxation [19], it provides a useful description of environmental effects in a simple way. To quantify the transfer of energy from a chosen site $k$ to the reaction center, we have phenomenologically introduced an irreversible decay process (with rate $\Gamma$ ) described by the operator $\hat{D}$, which is given by [20] $\hat{D}[\hat{\rho}]_{n m}=-\Gamma\{|k\rangle \times$ $\langle k|, \hat{\rho}\}_{n m}$, where $\{\cdots\}$ stands for the anticommutator.

Making use of Eq. (2), one can define a measure for the efficiency of energy transport as the population transferred to the reaction center, within a time $t$, as

$$
Q_{\text {eff }}=2 \Gamma \int_{0}^{t}\langle k|\hat{\rho}(s)| k\rangle d s .
$$

Equations (2) and (3) constitute the quantum equations, which have to be compared with the equations that will be obtained in the classical model.

For the classical case, we consider an ensemble of $N$ coupled harmonic oscillators, each with mass $M$ and frequency $\omega_{n}$. The temporal evolution of the system is described by a classical Hamiltonian, which in terms of the position $q_{n}$ and momentum $p_{n}$ of each oscillator reads as

$$
H_{S}=\sum_{n}\left(\frac{p_{n}^{2}}{2 M}+\frac{M \omega_{n}^{2}}{2} q_{n}^{2}\right)+\frac{1}{2} \sum_{n \neq m} K_{n m} q_{n} q_{m},
$$

where $K_{n m}$ stands for the coupling coefficient between the oscillators. By defining a new dimensionless complex amplitude [21], $\tilde{z}_{n}(t)=\tilde{q}_{n}(t)+i \tilde{p}_{n}(t)$, with $\tilde{q}_{n}=$ $\left(M \omega_{n} / 2 \hbar\right)^{1 / 2} q_{n}$ and $\tilde{p}_{n}=\left(2 \hbar M \omega_{n}\right)^{-1 / 2} p_{n}$, the Hamilton equations of motion of the system can be cast into a single equation:

$$
\frac{\partial \tilde{z}_{n}}{\partial t}=-i \omega_{n} \tilde{z}_{n}-i \sum_{m} \tilde{K}_{n m} \operatorname{Re}\left\{\tilde{z}_{m}\right\}
$$

$\operatorname{Re}\{\cdots\}$ stands for the real part of a complex number and $\tilde{K}_{n m}=K_{n m} /\left(M \sqrt{\omega_{n} \omega_{m}}\right)$.

To include environmental effects, we proceed in the same manner as in the construction of a Kubo oscillator $[22,23]$. For this, we assume that the frequency of each classical oscillator varies randomly as a stochastic process: $\omega_{n}(t)=\omega_{n}+\phi_{n}(t) . \omega_{n}$ is now the average frequency of the $n$th oscillator and $\phi_{n}(t)$ describes a Gaussian Markov process with zero average (Wiener process), i.e., $\left\langle\phi_{n}(t)\right\rangle=0$ and $\left\langle\phi_{n}(t) \phi_{m}\left(t^{\prime}\right)\right\rangle=\gamma_{n} \delta_{n m} \delta\left(t-t^{\prime}\right)$, where $\langle\cdots\rangle$ denotes stochastic averaging.

In Ref. [16], it has been shown that one can transform Eq. (5), within the framework of Itô calculus [24], into a classical master equation that describes the temporal dynamics of the system of coupled harmonic oscillators, when interaction with the surrounding environment is taken into account. To describe the transfer of excitation from the $k$ th oscillator to the reaction center, we extend this result, and introduce an irreversible decay process (with rate $\Gamma$ ), described by $\mathcal{D}[\sigma]_{n m}=-\Gamma\{|k\rangle\langle k|, \sigma\}_{n m}$, where $\sigma_{n m}=\left\langle\tilde{z}_{n} z_{m}^{*}\right\rangle$. In this way, we can write the classical master equation as

$$
\begin{aligned}
\frac{\partial \sigma_{n m}}{\partial t}= & \mathcal{H}[\sigma]_{n m}+\mathcal{L}[\sigma]_{n m}+\mathcal{D}[\sigma]_{n m} \\
& +\frac{i}{\hbar} \sum_{j}\left(V_{m j}\left\langle\tilde{z}_{j} \tilde{z}_{n}\right\rangle-V_{n j}\left\langle\tilde{z}_{j}^{*} \tilde{z}_{m}^{*}\right\rangle\right),
\end{aligned}
$$

with $V_{n m}=\tilde{K}_{n m} \hbar / 2$, and

$$
\begin{gathered}
\mathcal{H}[\sigma]_{n m}=-i\left(\omega_{n}-\omega_{m}\right) \sigma_{n m} \\
-\frac{i}{\hbar} \sum_{j}\left(V_{n j} \sigma_{j m}-V_{j m} \sigma_{n j}\right), \\
\mathcal{L}[\sigma]_{n m}=-\left[\frac{1}{2}\left(\gamma_{n}+\gamma_{m}\right)-\sqrt{\gamma_{n} \gamma_{m}} \delta_{n m}\right] \sigma_{n m} .
\end{gathered}
$$

The energy transfer efficiency within the ensemble of oscillators is then given by

$$
C_{\text {eff }}=2 \Gamma \int_{0}^{t} \bar{\sigma}_{k k}(s) d s .
$$

$\bar{\sigma}(t)=\sigma(t) / \sum_{n} \sigma_{n n}$ is the normalized classical density operator.

Equations (6) and (9) represent the classical equations, whose results have to be compared with their quantummechanical counterpart, Eqs. (2) and (3). To this end, we can make use of the site energies and coupling coefficients for the FMO complex of P. aestuarii [25]. The FMO is a pigment-protein complex that guides the energy from the light-harvesting chlorosomes to the reaction center in green sulfur bacteria [26,27]. It is a trimer of three identical subunits interacting weakly with each other. Each subunit is composed of seven bacteriochlorophyll-a (BChla) molecules embedded in a scaffolding of protein molecules, as shown in Fig. 1. The FMO complex is generally modeled 


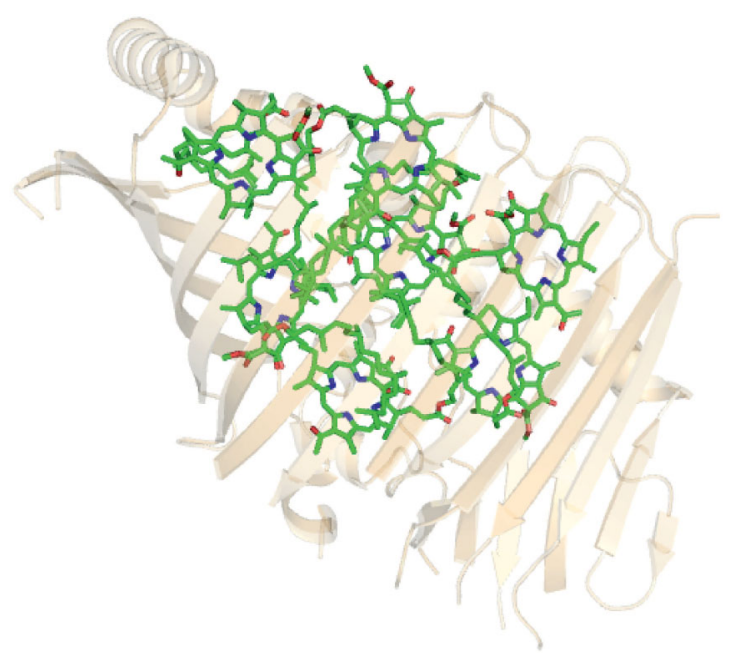

FIG. 1 (color online). Arrangement of the BChla molecules of a single unit of the Fenna-Matthews-Olson (FMO) complex. The figure was created using PyMOL [35], and is based on the Protein Data Bank entry 3ENI.

by a network of seven different sites, where the dynamics of a single excitation through the complex is governed by the specific values of the site energies $\left(\epsilon_{n}\right)$ and the coupling coefficients $\left(V_{n m}\right)$. In particular, we will use the values of the site energies and coupling coefficients for $P$. aestuarii, as stated in Tables 2 and 4 of Ref. [25]. The initial state of the system corresponds to a single excitation in site 1 . In the FMO, the BChl 3 is in the vicinity of the reaction center [25]. Thus, we take this site $(k=3)$ as the main excitation donor to the reaction center, with a transfer rate estimated to be $\Gamma=1 \mathrm{ps}^{-1}$ [28]. Furthermore, for the pure dephasing process, we consider that dephasing rates are the same for all sites $\left(\gamma=\gamma_{n}\right)$ and that the efficiency of energy transfer is limited by the finite excitation lifetime $(t \sim 1 \mathrm{~ns})$.

Figure 2 shows the efficiency of energy transfer as a function of the dephasing rate $\gamma$ obtained by means of the quantum equations (2) and (3). Notice that at low dephasing, i.e., with environment effects not considered, coherent

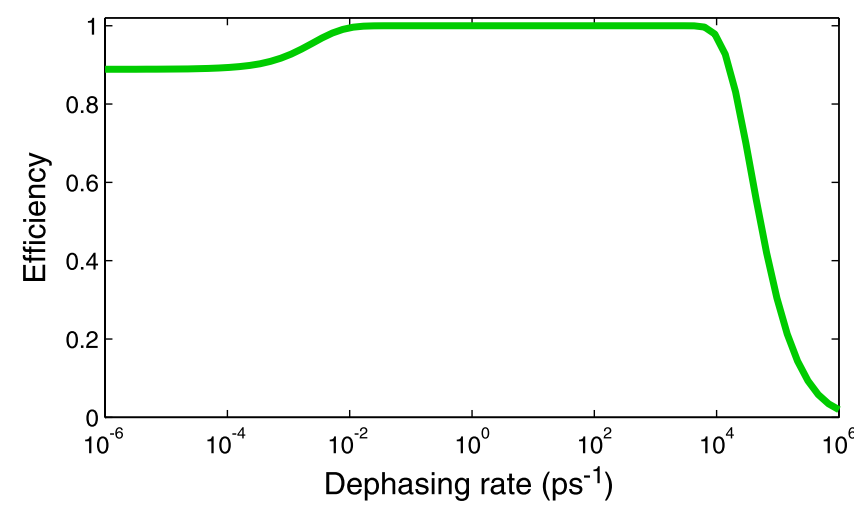

FIG. 2 (color online). Energy transfer efficiency as a function of the dephasing rate $\gamma$ obtained from the quantum-mechanical equations. evolution of the system leads to an efficiency of about $90 \%$. When increasing the dephasing, efficiency grows to almost $100 \%$, showing that the environment affects the system in such a way that it becomes more efficient for transferring energy to the reaction center. Finally, for stronger dephasing, efficiency drops rapidly and almost no energy is transferred to the reaction center. Qualitatively similar results have also been obtained for the case of the FMO complex of Chlorobium tepidum [13].

We now turn our attention to the case of the classical model by solving Eqs. (6) and (9). Figure 3 shows the efficiency of the energy transfer as a function of the dephasing rate. We observe that the same noise-assisted effect is also present in the purely classical model. For the sake of comparison, Fig. 3 also shows the solution of the quantummechanical model (dashed line). Notice that both solutions agree for dephasing rates up to $10^{3} \mathrm{ps}^{-1}$. However, for larger values of dephasing the quantum and classical solutions differ from each other. This is in agreement with the fact that both solutions are the same, provided that the condition $\gamma \ll \omega_{n}$ is satisfied [16].

Noise-assisted energy transport in disordered systems has been understood as the suppression of coherent quantum localization through noise, bringing the detuned quantum levels into resonance and thus facilitating the energy transfer $[13,15]$. Notwithstanding, the results presented here show that the same effect can also be found in purely classical systems. This implies that one can make use of such systems in order to simulate the intricate energy transfer mechanisms that take place in molecular aggregates, such as the photosynthetic FMO complex.

Recently, it has been suggested that classical $L C$ circuit oscillators (where $L$ stands for inductance and $C$ for capacitance) can be used to model coupled quantum twolevel systems [29]. Hence, one could devise an experimental apparatus comprising eight electrical oscillators with the eighth acting as the reaction center, which would be

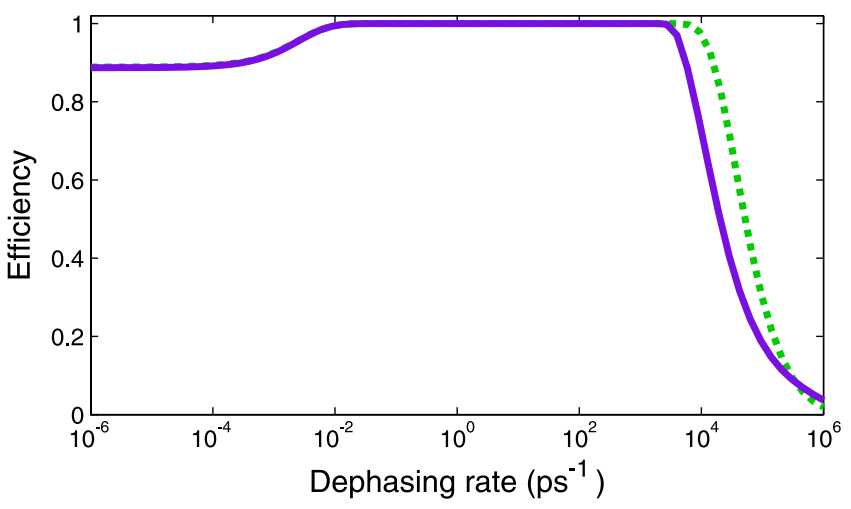

FIG. 3 (color online). Energy transfer efficiency as a function of the dephasing rate $\gamma$ obtained from the classical equations (solid line). For the sake of comparison, we have also included here the curve shown in Fig. 2, which corresponds to the solution of the quantum equations (dashed line). 
strongly coupled to one of the remaining oscillators. Then, by stochastically modulating the frequencies $\omega_{n}$, and properly controlling the noise intensity $\gamma_{n}$, one would be able to observe the noise-assisted energy transfer phenomenon by monitoring the signal present in the eighth oscillator. These classical simulations could be further used to compare with the recent experimental proposal of noise-assisted transport based on coupled quantum-optical cavities [30].

The concept of noise-assisted energy transport has been extensively used for describing the inner working of quantum and classical systems [31]. Along these lines, the particular enhancement effect described in this Letter might open a new research direction towards new methods for enhancing the efficiency of a myriad of energy transport systems that inevitably live in a noisy environment, from small-scale information and energy transfer systems in microwave and photonic circuits to long-distance highvoltage electrical lines. In this way, a specific feature initially conceived in a quantum scenario (environmentassisted energy transport) is shown to arise as well in a purely classical context, widening thus the scope of possible quantum-inspired technological applications.

To conclude, the search and demonstration of systems in which to observe quantum-mechanical effects with no classical counterpart is a subject of lively interest and debate [32-34]. Biological systems are not, in principle, a propitious scenario for the observation of quantum features, such as quantum superposition, interference, or entanglement. Nevertheless, one can always use a quantum perspective to describe any physical process, since everything follows the laws of quantum mechanics. This does not mean, however, that in certain cases a purely classical model may not similarly reproduce some of the results predicted by the full quantum-mechanical model, since classical physics emerge, after all, from quantum physics under many circumstances.

This work was supported by projects FIS2010-14831 and FET-Open Grant No. 255914 (PHORBITECH). This work has also been partially supported by Fundacio Privada Cellex Barcelona.

*roberto.leon@icfo.es †juanp.torres@icfo.es

[1] J. Frenkel, Phys. Rev. 37, 17 (1931).

[2] J. Franck and E. Teller, J. Chem. Phys. 6, 861 (1938).

[3] T. Förster, Modern Quantum Chemistry (Academic, New York, 1965).

[4] R.E. Blankenship, Molecular Mechanisms of Photosynthesis (Blackwell, Oxford, 2002).

[5] P. Ball, Nature (London) 474, 272 (2011).
[6] N. Lambert, Y.N. Chen, Y.C. Cheng, C. M. Li, G. Y. Chen, and F. Nori, Nat. Phys. 9, 10 (2013).

[7] G. S. Engel, T. R. Calhoun, E. L. Read, T.-K. Ahn, T. Mančal, Y.-C. Chen, R.E. Blankenship, and G. R. Fleming, Nature (London) 446, 782 (2007).

[8] G. Panitchayangkoon, D. Hayes, K. A. Fransted, J. R. Caram, E. Harel, J. Wen, R. E. Blankenship, and G. S. Engel, Proc. Natl. Acad. Sci. U.S.A. 107, 12766 (2010).

[9] E. Collini, C. Y. Wong, K. E. Wilk, P. M. G. Curmi, P. Brumer, and G.D. Scholes, Nature (London) 463, 644 (2010).

[10] A. Ishizaki and G. R. Fleming, J. Chem. Phys. 130, 234111 (2009).

[11] S. Hoyer, M. Sarovar, and K. B. Whaley, New J. Phys. 12, 065041 (2010).

[12] F. Fassioli and A. Olaya-Castro, New J. Phys. 12, 085006 (2010).

[13] P. Rebentrost, M. Mohseni, I. Kassal, S. Lloyd, and A. Aspuru-Guzik, New J. Phys. 11, 033003 (2009).

[14] M. Plenio and S. Huelga, New J. Phys. 10, 113019 (2008).

[15] I. Kassal and A. Aspuru-Guzik, New J. Phys. 14, 053041 (2012).

[16] A. Eisfeld and J. S. Briggs, Phys. Rev. E 85, 046118 (2012).

[17] J.S. Briggs and A. Eisfeld, Phys. Rev. E 83, 051911 (2011).

[18] H.-P. Breuer and F. Petruccione, The Theory of Open Quantum Systems (Oxford University, New York, 2002).

[19] A. Ishizaki and G. R. Fleming, J. Chem. Phys. 130, 234110 (2009).

[20] M. Scully and M. S. Zubairy, Quantum Optics (Cambridge University Press, Cambridge, England, 2006).

[21] F. Strocchi, Rev. Mod. Phys. 38, 36 (1966).

[22] R. Kubo, J. Math. Phys. (N.Y.) 4, 174 (1963).

[23] R. F. Fox, Phys. Rep. 48, 179 (1978).

[24] N. G. van Kampen, J. Stat. Phys. 24, 175 (1981).

[25] J. Adolphs and T. Renger, Biophys. J. 91, 2778 (2006).

[26] R. E. Fenna and B. W. Matthews, Nature (London) 258, 573 (1975).

[27] C. Sybesma and J. M. Olson, Proc. Natl. Acad. Sci. U.S.A. 49, 248 (1963).

[28] M. Mohseni, P. Rebentrost, S. Lloyd, and A. AspuruGuzik, J. Chem. Phys. 129, 174106 (2008).

[29] J.S. Briggs and A. Eisfeld, Phys. Rev. A 85, 052111 (2012).

[30] F. Caruso, N. Spagnolo, C. Vitelli, F. Sciarrino, and M. B. Plenio, Phys. Rev. A 83, 013811 (2011).

[31] P. Hänggi and F. Marchesoni, Rev. Mod. Phys. 81, 387 (2009).

[32] E. N. Zimanyi and R. J. Silbey, J. Chem. Phys. 133, 144107 (2010).

[33] W. H. Miller, J. Chem. Phys. 136, 210901 (2012).

[34] M. Tiersch, S. Popescu, and H. J. Briegel, Phil. Trans. R. Soc. A 370, 3771 (2012).

[35] Schrödinger LLC, The PyMOL Molecular Graphics System (version 1.4.1). 